\title{
Blow up of Coupled Nonlinear Klein-Gordon System with Distributed Delay, Strong Damping, and Source Terms
}

\author{
Djamel Ouchenane, ${ }^{1}$ Salah Mahmoud Boulaaras $\mathbb{D}^{2,3}{ }^{2,3}$ Asma Alharbi, ${ }^{2}$ and Bahri Cherif ${ }^{2}$ \\ ${ }^{1}$ Laboratory of Pure and Applied Mathematics, Amar Teledji Laghouat University, Algeria \\ ${ }^{2}$ Department of Mathematics, College of Sciences and Arts AlRas, Qassim University, Saudi Arabia \\ ${ }^{3}$ Laboratory of Fundamental and Applied Mathematics of Oran (LMFAO), University of Oran 1 Ahmed Ben Bella, Algeria
}

Correspondence should be addressed to Salah Mahmoud Boulaaras; s.boularas@qu.edu.sa

Received 19 July 2020; Revised 13 August 2020; Accepted 25 August 2020; Published 14 September 2020

Academic Editor: Fanglei Wang

Copyright (C) 2020 Djamel Ouchenane et al. This is an open access article distributed under the Creative Commons Attribution License, which permits unrestricted use, distribution, and reproduction in any medium, provided the original work is properly cited.

This present work deals with the blow up of the coupled Klein-Gordon system with strong damping, distributed delay, and source terms, under suitable conditions.

\section{Introduction}

In the present paper, we consider the following system:

$$
\left\{\begin{array}{l}
u_{t t}+m_{1} u^{2}-\Delta u-\omega_{1} \Delta u_{t}+\int_{0}^{t} g(t-s) \Delta u(s) d s+\mu_{1} u_{t}+\int_{\tau_{2}}^{\tau_{1}}\left|\mu_{2}(\varrho)\right| u_{t}(x, t-\mathrm{e}) d \mathrm{Q}=f_{1}(u, v), \quad(x, t) \in \Omega \times \mathbb{R}_{+}, v_{t t} m_{2} v^{2}-\Delta v-\omega_{2} \Delta v_{t}+\int_{0}^{t} h(t-s) \Delta v(s) d s+\mu_{3} v_{t}+\int_{\tau_{1}}^{\tau_{2}}\left|\mu_{4}(\mathrm{e})\right| v_{t}(x, t-\mathrm{e}) d \mathrm{Q}=f_{2}(u, v), \quad(x, t) \in \Omega \times \mathbb{R}_{+}, \\
u(x, t)=0, \quad v(x, t)=0, \quad x \in \partial \Omega, \\
u_{t}(x,-t)=f_{0}(x, t), \quad v_{t}(x,-t)=k_{0}(x, t) \quad(x, t) \in \Omega \times\left(0, \tau_{2}\right), \\
u(x, 0)=u_{0}(x), \quad u_{t}(x, 0)=u_{1}(x), \quad x \in \Omega \\
v(x, 0)=v_{0}(x), \quad v_{t}(x, 0)=v_{1}(x), \quad x \in \Omega
\end{array}\right.
$$

where

$$
\left\{\begin{array}{l}
f_{1}(u, v)=a_{1}|u+v|^{2(p+1)}(u+v)+b_{1}|u|^{p} \cdot u \cdot|v|^{p+2}, \\
f_{2}(u, v)=a_{1}|u+v|^{2(p+1)}(u+v)+b_{1}|v|^{p} \cdot v \cdot|u|^{p+2},
\end{array}\right.
$$

and $m_{1}, m_{2}, \omega_{1}, \omega_{2}, \mu_{1}, \mu_{3}, a_{1}, b_{1}>0$, and $\tau_{1}, \tau_{2}$ are the time delay with $0 \leq \tau_{1}<\tau_{2}$, and $\mu_{2}, \mu_{4}$ are a $L^{\infty}$ functions, and $g, h$ are differentiable functions.

Viscous materials are the opposite of flexible materials that have dissipate mechanical energy and the ability to store.
The mechanical properties of viscous materials are so important that we find them in many applications of natural sciences. Many authors have been concerned with this problem in recent decades.

If there is only one equation and if $w_{1}=0$, that is, for absence of $\Delta u_{t}$, and $\mu_{1}=\mu_{2}=0$. Our problem (1) has been studied by Berrimi and Messaoudi [1]. Using Galerkin's method they proved the result of local existence. They also made it clear that the local solution is global in time under suitable conditions and at the same rate of decaying (exponential or polynomial) of the kernel $g$. In addition, the authors themselves demonstrated that the dissipation can 
be deduced by the term viscous integral and that it is strong enough to stabilize the solution oscillations. Their results were also obtained under weaker conditions than those used by Cavalcanti et al. [2].

In [3], the authors considered the following problem:

$$
u_{t t}-\Delta u+\int_{0}^{t} g(t-s) \Delta u(s) d s+a(x) u_{t}+|u|^{\gamma}, u=0
$$

where the authors proved the exponential decay result. This subsequent result was improved by Berrimi et al. in [1], as they showed that the viscosity elastic dissipation alone is strong enough to stabilize the problem even with the exponential rate with respect to the kernel $g$ assumptions. In the case $\mu_{1} \neq 0$, in problem (1), Kafini and Messaoudi in [4] proved a blow up result for the following problem:

$$
\left\{\begin{array}{l}
u_{t t}-\Delta u+\int_{0}^{\infty} g(t-s) \Delta u(s) d s+u_{t}=|u|^{p-2} \cdot u, \quad(x, t) \in \mathbb{R}^{n} \times(0, \infty) \\
u(x, 0)=u_{0}(x), \quad u_{t}(x, 0)=u_{1}(x)
\end{array}\right.
$$

where $g$ satisfies

$$
\int_{0}^{\infty} g(s) d s<(2 p-4) /(2 p-3)
$$

The initial data was backed by negative energy as

$$
\int u_{0} u_{1} d x>0
$$

In [5], Song and Xue considered the following problem:

$$
\left\{\begin{array}{l}
u_{t t}-\Delta u+\int_{0}^{\infty} g(t-s) \Delta u(s) d s-\Delta u_{t}|u|^{p-2} \cdot u, \quad(x, t) \in \Omega \times(0, \infty) \\
u(x, 0)=u_{0}(x), \quad u_{t}(x, 0)=u_{1}(x)
\end{array}\right.
$$

where the authors showed that there were solutions of (7) with initial energy according to suitable assumptions on $g$. Moreover, they showed the blow up in a finite time. Then, the same authors in [6] continued to prove that there were solutions of (7) with positive initial energy that blow up in finite time. In [7], the author studied the following problem:

$$
\left\{\begin{array}{l}
u_{t t}-\Delta u-\omega \Delta u_{t}+\int_{0}^{t} g(t-s) \Delta u(s) d s+a\left|u_{t}\right|^{m-2} \cdot u_{t}=|u|^{p-2} \cdot u, \quad(x, t) \in \Omega(0, \infty) \\
u(x, 0)=u_{0}(x), \quad u_{t}(x, 0)=u_{1}(x), \quad x \in \Omega \\
u(x, t)=0, \quad x \in \partial \Omega
\end{array}\right.
$$

where they proved the exponential growth result under suitable assumptions. The authors in [8] studied the following problem:

$$
\left\{\begin{array}{l}
u_{t t}-\Delta u+\int_{0}^{\infty} g(s) \Delta u(t-s) d s-\varepsilon_{1} \Delta u_{t}+\varepsilon_{2} u_{t}\left|u_{t}\right|^{m-2}=\varepsilon_{3} u|u|^{p-2} \\
u(x, t)=0, \quad x \in \partial \Omega, t>0 \\
u(x, 0)=u_{0}(x), \quad u_{t}(x, 0)=u_{1}(x), \quad x \in \Omega
\end{array}\right.
$$

where they showed a blow up result if $p>m$ and established the global existence. In the coupled equation case, the authors in [9] studied the following system:

$$
\left\{\begin{array}{l}
u_{t t}-\Delta u+u_{t}\left|u_{t}\right|^{m-2}=f_{1}(u, v), \\
v_{t t}-\Delta v+v_{t}\left|v_{t}\right|^{r-2}=f_{2}(u, v)
\end{array}\right.
$$

with $f_{1}$ and $f_{2}$ nonlinear functions satisfying appropriate conditions. According to certain restrictions imposed on the initial data and parameters, they obtained numerous 
results on the existence of weak solutions. They obtained many results on the presence of weak solutions. In addition, by using the same techniques similar to that in [10] with negative initial, energy blows up for a finite period of time.

In [11], the authors have proved the solution of the problem:

$$
\left\{\begin{array}{l}
u_{t t}-\Delta u+\left(a|u|^{k}+b|v|^{l}\right) u_{t}\left|u_{t}\right|^{m-2}=f_{1}(u, v), \\
v_{t t}-\Delta v+\left(a|u|^{\theta}+b|v|^{9}\right) v_{t}\left|v_{t}\right|^{r-2}=f_{2}(u, v),
\end{array}\right.
$$

where under some restrictions on positive initial energy for certain conditions on the functions $f_{1}$ and $f_{2}$, the authors proved the blows up in finite time of solution.

The result of [11] has been extended by the authors in [12], where they studied the following system:

$$
\left\{\begin{array}{l}
u_{t t}-\Delta u+\int_{0}^{\infty} g(s) \Delta u(t-s) d s+\left(a|u|^{k}+b|v|^{l}\right) u_{t}\left|u_{t}\right|^{m-2}=f_{1}(u, v), \\
v_{t t}-\Delta v+\int_{0}^{\infty} h(s) \Delta v(t-s) d s+\left(a|u|^{\theta}+b|v|^{\varrho}\right) v_{t}\left|v_{t}\right|^{r-2}=f_{2}(u, v),
\end{array}\right.
$$

they proved that the solutions of a system of wave equations with degenerate damping, viscoelastic term and strong nonlinear sources acting in both equations at the same time are globally nonexisting provided that the initial data are sufficiently large in a bounded domain of $\Omega$.

As complement to these works, we are working to prove the blow up result with distributed delay of problem (1), under appropriate assumptions, and we prove these results using the energy method. In the following, let $c, c_{i}>0, i=1, \cdots, 12$.

The present paper is organized as follows. In Section 2, we give some necessarily assumptions for the main result. In Section 3, we prove the blow up result.

\section{Assumptions}

We consider the following suitable assumptions.

(A1) $g, h: \mathbb{R}_{+} \longrightarrow \mathbb{R}_{+}$are differentiable and decreasing functions such that

$$
g(t) \geq 0, \quad 1-\int_{0}^{\infty} g(s) d s=l_{1}>0
$$

$$
h(t) \geq 0, \quad 1-\int_{0}^{\infty} h(s) d s=l_{2}>0 .
$$

(A2) There exists a constants $\xi_{1}, \xi_{2}>0$ such that

$$
\begin{aligned}
& g^{\prime}(t) \leq-\xi_{1} g(t), \quad t \geq 0 \\
& h^{\prime}(t) \leq-\xi_{2} h(t), \quad t \geq 0 .
\end{aligned}
$$

(A3) $\mu_{2}, \mu_{4}:\left[\tau_{1}, \tau_{2}\right] \longrightarrow \mathbb{R}$ are a $L^{\infty}$ functions so that

$$
\begin{gathered}
\left(\frac{2 \delta-1}{2}\right) \int_{\tau_{1}}^{\tau_{2}}\left|\mu_{2}(\mathrm{\varrho})\right| d \mathrm{\varrho}<\mu_{1}, \quad \delta>\frac{1}{2}, \\
\left(\frac{2 \delta-1}{2}\right) \int_{\tau_{1}}^{\tau_{2}}\left|\mu_{4}(\mathrm{\varrho})\right| d \varrho<\mu_{3}, \quad \delta>\frac{1}{2} .
\end{gathered}
$$

\section{Blow up}

In this section, we obtain the proof of the blow up result of the solution of problem (1). First, of all in [13], we introduce the new variables

$$
\begin{aligned}
& y(x, \rho, \varrho, t)=u_{t}(x, t-\varrho \rho), \\
& z(x, \rho, \varrho, t)=v_{t}(x, t-\varrho \rho),
\end{aligned}
$$

then, we obtain

$$
\begin{aligned}
& \left\{\begin{array}{l}
\mathrm{\varrho} y_{t}(x, \rho, \varrho, t)+y_{\rho}(x, \rho, \varrho, t)=0, \\
y(x, 0, \varrho, t)=u_{t}(x, t),
\end{array}\right. \\
& \left\{\begin{array}{l}
\mathrm{\varrho} z_{t}(x, \rho, \varrho, t)+z_{\rho}(x, \rho, \varrho, t)=0, \\
z(x, 0, \varrho, t)=v_{t}(x, t) .
\end{array}\right.
\end{aligned}
$$

Let us denote by

$$
\text { gou }=\int_{\Omega} \int_{0}^{t} g(t-s)|u(t)-u(s)|^{2} d s d x
$$

Therefore, problem (1) get the following form:

$$
\left\{\begin{array}{l}
u_{t t}+m_{1} u^{2}-\Delta u-\omega_{1} \Delta u_{t}+\int_{0}^{t} g(t-s) \Delta u(s) d s+\mu_{1} u_{t}+\int_{\tau_{1}}^{\tau_{2}}\left|\mu_{2}(\varrho)\right| y(x, 1, \varrho, t) d \varrho=f_{1}(u, v), \quad x \in \Omega, t \geq 0, \\
v_{t t}+m_{2} v^{2}-\Delta v-\omega_{2} \Delta v_{t}+\int_{0}^{t} h(t-s) \Delta v(s) d s+\mu_{3} v_{t}+\int_{\tau_{1}}^{\tau_{2}}\left|\mu_{4}(\varrho)\right| z(x, 1, \varrho, t) d \varrho=f_{2}(u, v), \quad x \in \Omega, t \geq 0, \\
\varrho y_{t}(x, \rho, \varrho, t)+y_{p}(x, \rho, \varrho, t)=0, \\
\varrho z_{t}(x, \rho, \varrho, t)+z_{p}(x, \rho, \varrho, t)=0,
\end{array}\right.
$$


with initial and boundary conditions

$$
\left\{\begin{array}{l}
u(x, t)=0, \quad v(x, t)=0, \quad x \in \partial \Omega, \\
y(x, \rho, \varrho, 0)=f_{0}(x, \varrho \rho), \quad z(x, \rho, \varrho, 0)=k_{0}(x, \varrho \rho), \\
u(x, 0)=u_{0}(x), \quad u_{t}(x, 0)=u_{1}(x), \\
v(x, 0)=v_{0}(x), \quad v_{t}(x, 0)=v_{1}(x),
\end{array}\right.
$$

where

$$
(x, \rho, \varrho, t) \in \Omega \times(0,1) \times\left(\tau_{1}, \tau_{2}\right) \times(0, \infty) .
$$

Theorem 1. Assume (14), (16), and (17) hold. Let

$$
\left\{\begin{array}{l}
-1<p<\frac{4-n}{n-2}, \quad n \geq 3 \\
p \geq-1, \quad n=1,2 .
\end{array}\right.
$$

For any initial data,

$$
\left(u_{o}, u_{1}, v_{0}, v_{1}, f_{0}, k_{0}\right) \in \mathscr{H}
$$

where

$$
\begin{aligned}
\mathscr{H}= & H_{0}^{1}(\Omega) \times L^{2}(\Omega) \times H_{0}^{1}(\Omega) \times L^{2}(\Omega) \\
& \times L^{2}\left(\Omega \times(0,1) \times\left(\tau_{1}, \tau_{2}\right)\right) \times L^{2}\left(\Omega \times(0,1) \times\left(\tau_{1}, \tau_{2}\right)\right),
\end{aligned}
$$

then, problem (22) has a unique solution

$$
u \in C([0, T] ; \mathscr{H})
$$

for some $T>0$.

Lemma 2. There exists a function $F(u, v)$ such that

$$
\begin{aligned}
F(u, v) & =\frac{1}{2(\rho+2)}\left[u f_{1}(u, v)+v f_{2}(u, v)\right] \\
& =\frac{1}{2(\rho+2)}\left[a_{1}|u+v|^{2(p+2)}+2 b_{1}|u v|^{p+2}\right] \geq 0
\end{aligned}
$$

where

$$
\begin{aligned}
& \frac{\partial F}{\partial u}=f_{1}(u, v), \\
& \frac{\partial F}{\partial v}=f_{2}(u, v),
\end{aligned}
$$

we take $a_{1}=b_{1}=1$ for convenience.
Lemma 3. (see [12]). There exist two positive constants $c_{0}$ and $c_{1}$ such that

$$
\begin{aligned}
& \frac{c_{0}}{2(\rho+2)}\left(|u|^{2(p+2)}+|v|^{2(p+2)}\right) \\
& \quad \leq F(u, v) \leq \frac{c_{1}}{2(\rho+2)}\left(|u|^{2(p+2)}+|v|^{2(p+2)}\right) .
\end{aligned}
$$

We define the energy functional (see, e.g., [14-16] and reference therein).

Lemma 4. Assume (14), (16), (17), and (25) hold, let (u, v, $y, z)$ be a solution of (22), then $E(t)$ is nonincreasing, that is,

$$
\begin{aligned}
E(t)= & \frac{1}{2}\left\|u_{t}\right\|_{2}^{2}+\frac{1}{2}\left\|v_{t}\right\|_{2}^{2}+\frac{m_{1}}{2}\|u\|_{2}^{2}+\frac{m_{2}}{2}\|v\|_{2}^{2} \\
& +\frac{1}{2} l_{1}\|\nabla u\|_{2}^{2}+\frac{1}{2} l_{2}\|\nabla v\|_{2}^{2}+\frac{1}{2}(g o \nabla u)+\frac{1}{2}(h o \nabla u) \\
& +\frac{1}{2} K(y, z)-\int_{\Omega} F(u, v) d x,
\end{aligned}
$$

satisfies

$$
\begin{aligned}
E^{\prime}(t) \leq & -c_{3}\left\{\left\|u_{t}\right\|_{2}^{2}+\left\|v_{t}\right\|_{2}^{2}+\|u\|_{2}^{2}+\|v\|_{2}^{2}\right. \\
& +\int_{\Omega}^{\tau_{2}} \int_{\tau_{1}}^{\tau_{2}}\left|\mu_{2}(\mathrm{\varrho})\right| y^{2}(x, 1, \varrho, t) d \varrho d x \\
& \left.+\int_{\Omega} \int_{\tau_{1}}^{\tau_{2}}\left|\mu_{4}(\mathrm{\varrho})\right| z^{2}(x, 1, \varrho, t) d \mathrm{\varrho} d x\right\} \leq 0,
\end{aligned}
$$

where

$$
\begin{aligned}
K(y, z)= & \int_{\Omega} \int_{0}^{1} \int_{\tau_{1}}^{\tau_{2}} \mathrm{\varrho}\left\{\left|\mu_{2}(\mathrm{\varrho})\right| y^{2}(x, \rho,, \mathrm{\varrho}, t)\right. \\
& \left.+\left|\mu_{4}(\mathrm{\varrho})\right| z^{2}(x, \rho,, \mathrm{\varrho}, t) d \mathrm{\varrho} d \rho d x\right\} .
\end{aligned}
$$

Proof. By multiplying $(3.4)_{1},(3.4)_{2}$ by $u_{t}, v_{t}$ and integrating over $\Omega$, we get

$$
\begin{aligned}
\frac{d}{d t}\left\{\frac{1}{2}\left\|u_{t}\right\|_{2}^{2}+\frac{1}{2}\left\|v_{t}\right\|_{2}^{2}+\frac{m_{1}}{2}\|u\|_{2}^{2}+\frac{m_{2}}{2}\|v\|_{2}^{2}+\frac{1}{2} l_{1}\|\nabla u\|_{2}^{2}\right. \\
\left.\quad+\frac{1}{2} l_{2}\|\nabla v\|_{2}^{2}+\frac{1}{2}(g o \nabla u)+\frac{1}{2}(h o \nabla u)-\int_{\Omega} F(u, v) d x\right\} \\
=-\mu_{1}\left\|u_{t}\right\|_{2}^{2}-m_{1}\|u\|_{2}^{2}-\int_{\Omega} u_{t} \int_{\tau_{1}}^{\tau_{2}}\left|\mu_{2}(\mathrm{\varrho})\right| y(x, 1, \varrho, t) d \varrho d x \\
-\mu_{3}\left\|v_{t}\right\|_{2}^{2}-m_{2}\|v\|_{2}^{2}-\int_{\Omega} v_{t} \int_{\tau_{1}}^{\tau_{2}}\left|\mu_{4}(\mathrm{\varrho})\right| z(x, 1, \varrho, t) d \mathrm{\varrho} d x \\
+\frac{1}{2}\left(g^{\prime} o \nabla u\right)-\frac{1}{2} g(t)\|\nabla u\|_{2}^{2}-\omega_{1}\left\|\nabla u_{t}\right\|_{2}^{2}+\frac{1}{2}\left(h^{\prime} o \nabla u\right) \\
\quad-\frac{1}{2} h(t)\|\nabla v\|_{2}^{2}-\omega_{2}\left\|\nabla v_{t}\right\|_{2}^{2},
\end{aligned}
$$


and, from $(3.4)_{3},(3.4)_{4}$, we have

$$
\begin{aligned}
\frac{d}{d t} \frac{1}{2} \int_{\Omega} \int_{0}^{1} \int_{\tau_{1}}^{\tau_{2}} \mathrm{\varrho}\left|\mu_{2}(\mathrm{\varrho})\right| y^{2}(x, \rho, \mathrm{\varrho}, t) d \mathrm{\varrho} d \rho d x \\
=-\frac{1}{2} \int_{\Omega} \int_{0}^{1} \int_{\tau_{1}}^{\tau_{2}} 2\left|\mu_{2}(\mathrm{\varrho})\right| y y_{\rho} d \mathrm{\varrho} d \rho d x, \\
\quad+\frac{1}{2} \int_{\Omega} \int_{\tau_{1}}^{\tau_{2}}\left|\mu_{2}(\mathrm{\varrho})\right| y^{2}(x, 0, \mathrm{\varrho}, t) d \mathrm{\varrho} d x \\
\quad-\frac{1}{2} \int_{\Omega} \int_{\tau_{1}}^{\tau_{2}}\left|\mu_{2}(\mathrm{\varrho})\right| y^{2}(x, 1, \mathrm{\varrho}, t) d \mathrm{\varrho} d x, \\
=\frac{1}{2}\left(\int_{\tau_{1}}^{\tau_{2}} \mid \mu_{2}(\mathrm{\varrho}) d \mathrm{\varrho}\right) \|\left. u_{t}\right|_{2} ^{2} \\
\quad-\frac{1}{2} \int_{\Omega}^{\tau_{2}} \int_{\tau_{1}}^{\tau_{2}}\left|\mu_{2}(\mathrm{\varrho})\right| y^{2}(x, 1, \mathrm{\varrho}, t) d \mathrm{\varrho} d x, \\
\frac{d}{d t} \frac{1}{2} \int_{\Omega} \int_{0}^{1} \int_{\tau_{1}}^{\tau_{2}} \mathrm{\varrho}\left|\mu_{4}(\mathrm{\varrho})\right| z^{2}(x, \rho, \mathrm{\varrho}, t) d \mathrm{\varrho} d \rho d x \\
=-\frac{1}{2} \int_{\Omega} \int_{0}^{1} \int_{\tau_{1}}^{\tau_{2}} 2\left|\mu_{4}(\mathrm{\varrho})\right| z z \rho d \mathrm{\varrho} d \rho d x, \\
\quad+\frac{1}{2} \int_{\Omega}^{\tau_{2}} \int_{\tau_{1}}^{\tau_{2}}\left|\mu_{4}(\mathrm{\varrho})\right| z^{2}(x, 0, \mathrm{\varrho}, t) d \mathrm{\varrho} d x \\
\left.\quad-\frac{1}{2} \int_{\Omega}^{\tau_{2}} \int_{\tau_{1}}^{\tau_{2}}\left|\mu_{4}(\mathrm{\varrho})\right| z^{2}(x, 1, \mathrm{\varrho}, t) d \mathrm{\varrho} d x, \mid \mu_{4}(\mathrm{\varrho}) d \mathrm{\varrho}\right) \|\left. v_{t}\right|_{2} ^{2} \\
\quad-\frac{1}{2} \int_{\Omega}^{\tau_{2}} \int_{\tau_{1}}^{\tau_{2}}\left|\mu_{4}(\mathrm{\varrho})\right| z^{2}(x, 1, \mathrm{\varrho}, t) d \mathrm{\varrho} d x, \\
=
\end{aligned}
$$

then, we get

$$
\begin{aligned}
\frac{d}{d t} E( & t) \\
= & -\mu_{1}\left\|u_{t}\right\|_{2}^{2}-m_{1}\|u\|_{2}^{2}-\int_{\Omega} \int_{\tau_{1}}^{\tau_{2}}\left|\mu_{2}(\mathrm{\varrho})\right| u_{t} y(x, 1, \mathrm{\varrho}, t) d \mathrm{\varrho} d x \\
& +\frac{1}{2}\left(g^{\prime} o \nabla u\right)-\frac{1}{2} g(t)\|\nabla u\|_{2}^{2}-\omega_{1}\left\|\nabla u_{t}\right\|_{2}^{2} \\
& +\frac{1}{2}\left(\int_{\tau_{1}}^{\tau_{2}}\left|\mu_{2}(\mathrm{\varrho}) d \mathrm{\varrho}\left\|u_{t}\right\|_{2}^{2}-\frac{1}{2} \int_{\Omega} \int_{\tau_{1}}^{\tau_{2}}\right| \mu_{2}(\mathrm{\varrho}) \mid y^{2}(x, 1, \mathrm{\varrho}, t) d \mathrm{\varrho} d x\right. \\
& -\mu_{3}\left\|v_{t}\right\|_{2}^{2}-m_{2}\|v\|_{2}^{2}-\int_{\Omega}^{\tau_{2}}\left|\mu_{\tau_{1}}(\mathrm{\varrho})\right| v_{t} z(x, 1, \mathrm{\varrho}, t) d \mathrm{\varrho} d x \\
& +\frac{1}{2}\left(h^{\prime} o \nabla v\right)-\frac{1}{2} h(t)\|\nabla v\|_{2}^{2}-\omega_{2}\left\|\nabla v_{t}\right\|_{2}^{2} \\
& +\frac{1}{2}\left(\int_{\tau_{1}}^{\tau_{2}} \mid \mu_{4} \mathrm{\varrho} d \mathrm{\varrho}\right)\left\|v_{t}\right\|_{2}^{2}-\frac{1}{2} \int_{\Omega} \int_{\tau_{1}}^{\tau_{2}}\left|\mu_{4}(\mathrm{\varrho})\right| z^{2}(x, 1, \mathrm{\varrho}, t) d \mathrm{\varrho} d x .
\end{aligned}
$$

By (35)-(37), we get (32).

And by using Young's inequality, (14), (16), and (17) in (38), we obtain (33).
Now, we define the functional

$$
\begin{aligned}
\mathbb{H}(t)= & -E(t)=-\frac{1}{2}\left\|u_{t}\right\|_{2}^{2}-\frac{1}{2}\left\|v_{t}\right\|_{2}^{2}-\frac{m_{1}}{2}\|u\|_{2}^{2}-\frac{m_{2}}{2}\|v\|_{2}^{2} \\
& -\frac{1}{2} l_{1}\|\nabla u\|_{2}^{2}-\frac{1}{2} l_{2}\|\nabla v\|_{2}^{2}-\frac{1}{2}(g o \nabla u)-\frac{1}{2}(h o \nabla u) \\
& -\frac{1}{2} K(y, z)+\frac{1}{2(p+2)}\left[\|u+v\|_{2(p+2)}^{2(p+2)}+2\|u v\|_{(p+2)}^{(p+2)}\right] .
\end{aligned}
$$

Theorem 5. Assume (14)-(17) and (25) hold. Assume further that $E(0)<0$, then the solution of problem (22) blow up in finite time.

Proof. From (32), we have

$$
E(t) \leq E(0) \leq 0
$$

Therefore,

$$
\begin{aligned}
\mathbb{H}^{\prime}(t)= & -E^{\prime}(t) \\
\geq & c_{3}\left(\left\|u_{t}\right\|_{2}^{2}+\|u\|_{2}^{2}+\int_{\Omega} \int_{\tau_{1}}^{\tau_{2}}\left|\mu_{2}(\varrho)\right| y^{2}(x, 1, \varrho, t) d \varrho d x\right. \\
& \left.+\left\|v_{t}\right\|_{2}^{2}+\|v\|_{2}^{2}+\int_{\Omega} \int_{\tau_{1}}^{\tau_{2}}\left|\mu_{4}(\varrho)\right| z^{2}(x, 1, \varrho, t) d \varrho d x\right),
\end{aligned}
$$

hence,

$$
\mathbb{H}^{\prime}(t) \geq c_{3} \int_{\Omega} \int_{\tau_{1}}^{\tau_{2}}\left|\mu_{2}(\varrho)\right| y^{2}(x, 1, \varrho, t) d \varrho d x \geq 0
$$

$$
\begin{aligned}
\mathbb{H}^{\prime}(t) & \geq c_{3} \int_{\Omega} \int_{\tau_{1}}^{\tau_{2}}\left|\mu_{2}(\mathrm{\varrho})\right| z^{2}(x, 1, \mathrm{\varrho}, t) d \mathrm{\varrho} d x \geq 0 \\
0 & \leq \mathbb{H}(0) \mathbb{H}(t) \leq \frac{1}{2(p+2)}\left[\|u+v\|_{2(p+2)}^{2(p+2)}+2\|u v\|_{p+2}^{p+2}\right] \\
& \leq \frac{c_{1}}{2(p+2)}\left[\|u\|_{2(p+2)}^{2(p+2)}+\|v\|_{2(p+2)}^{2(p+2)}\right]
\end{aligned}
$$

We set

$$
\begin{aligned}
\mathscr{K}(t)= & \mathrm{H}^{1-\alpha}+\varepsilon \int_{\Omega}\left(u u_{t}+v v_{t}\right) d x+\frac{3}{2} \int_{\Omega}\left(\mu_{1} u^{2}+\mu_{3} v^{2}\right) d x \\
& +\frac{\varepsilon}{2} \int_{\Omega}\left(\omega_{1}(\nabla u)^{2}+\omega_{2}(\nabla u)^{2}\right) d x
\end{aligned}
$$

where $\varepsilon>0$ to be assigned later and

$$
0<\alpha<\frac{2 p+2}{4(p+2)}<1 .
$$


By multiplying $(3.4)_{1},(3.4)_{2}$ by $u, v$ and with a derivative of (45), we get

$$
\begin{aligned}
\mathscr{K}^{\prime}(t)= & (1-\alpha) \mathbb{H}^{-\alpha} \mathbb{H}^{\prime}(t)+\varepsilon\left(\left\|u_{t}\right\|_{2}^{2}+\left\|v_{t}\right\|_{2}^{2}+\|u\|_{2}^{2}+\|v\|_{2}^{2}\right) \\
& -\varepsilon\left(\|\nabla u\|_{2}^{2}+\|\nabla v\|_{2}^{2}\right)+\varepsilon \int_{\Omega} \nabla u \int_{0}^{1} g(t-s) \nabla u(s) d s d x \\
& +\varepsilon \int_{\Omega} \nabla v \int_{0}^{1} h(t-s) \nabla v(s) d s d x \\
& -\varepsilon \int_{\Omega} \int_{\tau_{1}}^{\tau_{2}}\left|\mu_{2}(\mathrm{\varrho})\right| u y(x, 1, \varrho, t) d \varrho d x \\
& -\varepsilon \int_{\Omega} \int_{\tau_{1}}^{\tau_{2}}\left|\mu_{4}(\mathrm{\varrho})\right| v z(x, 1, \varrho, t) d \varrho d x \\
& +\varepsilon\left[\|u+v\|_{2(p+2)}^{2(p+2)}+2\|u v\|_{p+2}^{p+2}\right] .
\end{aligned}
$$

Using Young's inequality, we get

$$
\begin{gathered}
\varepsilon \int_{\Omega} \int_{\tau_{1}}^{\tau_{2}}\left|\mu_{2}(\mathrm{\varrho})\right| u y(x, 1, \mathrm{\varrho}, t) d \mathrm{\varrho} d x \\
\leq \varepsilon\left\{\delta_{1}\left(\int_{\tau_{1}}^{\tau_{2}}\left|\mu_{2}(\mathrm{\varrho})\right| d \mathrm{\varrho}\right)\|u\|_{2}^{2}\right. \\
\left.+\frac{1}{4 \delta_{1}} \int_{\Omega} \int_{\tau_{1}}^{\tau_{2}}\left|\mu_{2}(\mathrm{\varrho})\right| y^{2}(x, 1, \mathrm{\varrho}, t) d \mathrm{\varrho} d x\right\}, \\
\varepsilon \int_{\Omega} \int_{\tau_{1}}^{\tau_{2}}\left|\mu_{4}(\mathrm{\varrho})\right| v z(x, 1, \mathrm{\varrho}, t) d \mathrm{\varrho} d x \\
\leq \varepsilon\left\{\delta_{2}\left(\int_{\tau_{1}}^{\tau_{2}}\left|\mu_{4}(\mathrm{\varrho})\right| d \mathrm{\varrho}\right)\|u\|_{2}^{2}\right. \\
\left.+\frac{1}{4 \delta_{2}} \int_{\Omega} \int_{\tau_{1}}^{\tau_{2}}\left|\mu_{4}(\mathrm{\varrho})\right| z^{2}(x, 1, \mathrm{\varrho}, t) d \mathrm{\varrho} d x\right\},
\end{gathered}
$$

and we have

$$
\begin{aligned}
& \varepsilon \int_{0}^{t} g(t-s) d s \int_{\Omega} \nabla u \cdot \nabla u(s) d x d s \\
& =\varepsilon \int_{0}^{t} g(t-s) d s \int_{\Omega} \nabla u \cdot(\nabla u(s)-\nabla u(t)) d x d s \\
& \quad+\varepsilon \int_{0}^{t} g(s) d s\|\nabla u\|_{2}^{2} \geq \frac{\varepsilon}{2} \int_{0}^{t} g(s) d s\|\nabla u\|_{2}^{2}-\frac{\varepsilon}{2}(g o \nabla u), \\
& \varepsilon \int_{0}^{t} h(t-s) d s \int_{\Omega} \nabla v \cdot \nabla v(s) d x d s \\
& \quad \varepsilon \int_{0}^{t} h(t-s) d s \int_{\Omega} \nabla v \cdot(\nabla v(s)-\nabla v(t)) d x d s \\
& \quad+\varepsilon \int_{0}^{t} h(s) d s\|\nabla v\|_{2}^{2} \geq \frac{\varepsilon}{2} \int_{0}^{t} h(s) d s\|\nabla v\|_{2}^{2}-\frac{\varepsilon}{2}(h o \nabla v) .
\end{aligned}
$$

We obtain, from (47),

$$
\begin{aligned}
\mathscr{K}^{\prime}(t) \geq & (1-\alpha) \mathbb{H}^{-\alpha} \mathbb{H}^{\prime}(t)+\varepsilon\left(\left\|u_{t}\right\|_{2}^{2}+\left\|u_{t}\right\|_{2}^{2}+\|u\|_{2}^{2}+\|v\|_{2}^{2}\right) \\
& -\varepsilon\left(\left(1-\frac{1}{2} \int_{0}^{t} g(s) d s\right)\|\nabla u\|_{2}^{2}\right. \\
& \left.+\left(1-\frac{1}{2} \int_{0}^{t} h(s) d s\right)\|\nabla v\|_{2}^{2}\right) \\
& -\varepsilon \delta_{1}\left(\int_{\tau_{1}}^{\tau_{2}}\left|\mu_{2}(\varrho)\right| d \varrho\right)\|u\|_{2}^{2} \\
& -\varepsilon \delta_{2}\left(\int_{\tau_{1}}^{\tau_{2}}\left|\mu_{4}(\varrho)\right| d \varrho\right)\|v\|_{2}^{2}-\frac{\varepsilon}{2}(g o \nabla u) \\
& -\frac{\varepsilon}{4 \delta_{1}} \int_{\Omega}^{\tau_{2}} \int_{\tau_{1}}^{\tau_{2}}\left|\mu_{2}(\varrho)\right| y^{2}(x, 1, \varrho, t) d \varrho d x-\frac{\varepsilon}{2}(h o \nabla v) \\
& -\frac{\varepsilon}{4 \delta_{2}} \int_{\Omega}^{\tau_{2}} \int_{\tau_{1}}^{\tau_{2}}\left|\mu_{4}(\varrho)\right| z^{2}(x, 1, \varrho, t) d \varrho d x \\
& +\varepsilon\left[\|u+v\|_{2(p+2)}^{2(p+2)}+2\|u v\|_{p+2}^{p+2}\right] .
\end{aligned}
$$

Therefore, using (43) and by setting $\delta_{1}, \delta_{1}$ so that, $1 / 4 \delta_{1}$ $c_{3}=\kappa \mathbb{H}^{-\alpha}(t) / 2$ and $1 / 4 \delta_{2} c_{3}=\kappa \mathbb{H}^{-\alpha}(t) / 2$, substituting in (50), we get

$$
\begin{aligned}
\mathscr{K}^{\prime}(t) \geq[ & (1-\alpha)-\varepsilon \kappa] \mathbb{H}^{-\alpha} \mathbb{H}^{\prime}(t) \\
& +\varepsilon\left(\left\|u_{t}\right\|_{2}^{2}+\left\|v_{t}\right\|_{2}^{2}+\|u\|_{2}^{2}+\|v\|_{2}^{2}\right) \\
& -\varepsilon\left[\left(1-\frac{1}{2} \int_{0}^{t} g(s) d s\right)\right]\|\nabla u\|_{2}^{2} \\
& -\varepsilon\left[\left(1-\frac{1}{2} \int_{0}^{t} h(s) d s\right)\right]\|\nabla v\|_{2}^{2} \\
& -\varepsilon \frac{\mathbb{H}^{\alpha}(t)}{2 c_{3} \kappa}\left(\int_{\tau_{1}}^{\tau_{2}}\left|\mu_{2}(\mathrm{\varrho})\right| d \mathrm{\varrho}\right)\|u\|_{2}^{2}-\frac{\varepsilon}{2}(g o \nabla u) \\
& -\varepsilon \frac{\mathrm{H}^{\alpha}(t)}{2 c_{3} \kappa}\left(\int_{\tau_{1}}^{\tau_{2}}\left|\mu_{4}(\mathrm{\varrho})\right| d \varrho\right)\|v\|_{2}^{2}-\frac{\varepsilon}{2}(h o \nabla u) \\
& +\varepsilon\left[\|u+v\|_{2(p+2)}^{2(p+2)}+2\|u v\|_{p+2}^{p+2}\right] .
\end{aligned}
$$

For $0<a<1$, from (39),

$$
\begin{aligned}
\varepsilon[\| u+ & \left.v\left\|_{2(p+2)}^{2(p+2)}+2\right\| u v \|_{p+2}^{p+2}\right] \\
= & \varepsilon a\left[\|u+v\|_{2(p+2)}^{2(p+2)}+2\|u v\|_{p+2}^{p+2}\right]+\varepsilon 2(p+2)(1-a) \mathrm{H}(t) \\
& +\varepsilon(p+2)(1-a)\left(\left\|u_{t}\right\|_{2}^{2}+\left\|v_{t}\right\|_{2}^{2}\right)+\varepsilon(p+2)(1-a) \\
& \cdot\left(1-\int_{0}^{t} g(s) d s\|\nabla u\|_{2}^{2}+\varepsilon(p+2)(1-a)\right. \\
& \cdot\left(1-\int_{0}^{t} h(s) d s\|\nabla v\|_{2}^{2}-\varepsilon(p+2)(1-a)(g o \nabla u)\right. \\
& -\varepsilon(p+2)(1-a)(h o \nabla v)+\varepsilon(p+2)(1-a) K(y, z)
\end{aligned}
$$


substituting in (51), we get

$$
\begin{aligned}
\mathscr{K}^{\prime}(t) \geq & {[(1-\alpha)-\varepsilon \kappa] \mathbb{H}^{-\alpha} \mathbb{H}^{\prime}(t) } \\
& +\varepsilon[(p+2)(1-\alpha)+1]\left(\left\|u_{t}\right\|_{2}^{2}+\left\|v_{t}\right\|_{2}^{2}+\|u\|_{2}^{2}+\|v\|_{2}^{2}\right) \\
& +\varepsilon\left[(p+2)(1-\alpha)\left(1-\int_{0}^{t} g(s) d s\right)\right. \\
& \left.-\left(1-\frac{1}{2} \int_{0}^{t} g(s) d s\right)\right]\|\nabla u\|_{2}^{2} \\
& +\varepsilon\left[(p+2)(1-\alpha)\left(1-\int_{0}^{t} h(s) d s\right)\right. \\
& \left.-\left(1-\frac{1}{2} \int_{0}^{t} h(s) d s\right)\right]\|\nabla v\|_{2}^{2} \\
& -\varepsilon \frac{H^{\alpha}(t)}{2 c_{3} \kappa}\left(\int_{\tau_{1}}^{\tau_{2}}\left|\mu_{2}(\varrho)\right| d \varrho\right)\|u\|_{2}^{2} \\
& -\varepsilon \frac{H^{\alpha}(t)}{2 c_{3} \kappa}\left(\int_{\tau_{1}}^{\tau_{2}}\left|\mu_{4}(\varrho)\right| d \varrho\right)\|v\|_{2}^{2} \\
& +\varepsilon(p+2)(1-\alpha) K(y, z) \\
& +\varepsilon\left[(p+2)(1-\alpha)-\frac{1}{2}\right](g o \nabla u+h o \nabla v) \\
& +\varepsilon a\left[\|u+v\|_{2(p+2)}^{2(p+2)}+2\|u v\|_{p+2}^{p+2}\right] \\
& +\varepsilon 2(p+2)(1-\alpha) \mathbb{H}(t) .
\end{aligned}
$$

Since (25) hold, we obtain by using (44) and (46)

$$
\begin{aligned}
& \mathbb{H}^{\alpha}(t)\|u\|_{2}^{2} \leq c_{4}\left(\|u\|_{2(p+2)}^{2 \alpha(p+2)+2}+\|v\|_{2(p+2)}^{2 \alpha(p+2)+2}\|u\|_{2}^{2}\right), \\
& \mathbb{H}^{\alpha}(t)\|v\|_{2}^{2} \leq c_{5}\left(\|v\|_{2(p+2)}^{2 \alpha(p+2)+2}+\|u\|_{2(p+2)}^{2 \alpha(p+2)+2}\|v\|_{2}^{2}\right),
\end{aligned}
$$

for some positive constants $c_{4}, c_{5}$. By using (46) and the algebraic inequality,

$B^{\theta} \leq(B+1) \leq\left(1+\frac{1}{b}\right)(B+b), \quad \forall B>0, \quad 0<\theta<1, \quad b>0$,

we have, $\forall t>0$

$$
\begin{aligned}
& \|u\|_{2(p+2)}^{2 \alpha(p+2)+2} \leq d\left(\|u\|_{2(p+2)}^{2(p+2)}+\mathbb{H}(0)\right) \leq d\left(\|u\|_{2(p+2)}^{2(p+2)}+\mathbb{H}(t)\right) \\
& \|v\|_{2(p+2)}^{2 \alpha(p+2)+2} \leq d\left(\|v\|_{2(p+2)}^{2(p+2)}+\mathbb{H}(t)\right) \leq d\left(\|v\|_{2(p+2)}^{2(p+2)}+\mathbb{H}(t)\right),
\end{aligned}
$$

where $d=1+(1 / \mathbb{H}(0))$. Also, since

$$
(x+y)^{\gamma} \leq C\left(x^{\gamma}+y^{\gamma}\right), \quad \forall x, y>0, \quad \gamma>0,
$$

we conclude

$$
\begin{aligned}
\|v\|_{2(p+2)}^{2 \alpha(p+2)}\|u\|_{2}^{2} & \leq c_{6}\left(\|v\|_{2(p+2)}^{2(p+2)}+\|u\|_{2}^{2(p+2)}\right) \\
\leq c_{7}\left(\|v\|_{2(p+2)}^{2(p+2)}+\|u\|_{2(p+2)}^{2(p+2)}\right), & \\
\|u\|_{2(p+2)}^{2 \alpha(p+2)}\|v\|_{2}^{2} & \leq c_{8}\left(\|u\|_{2(p+2)}^{2(p+2)}+\|v\|_{2}^{2(p+2)}\right) \\
& \leq c_{9}\left(\|u\|_{2(p+2)}^{2(p+2)}+\|v\|_{2(p+2)}^{2(p+2)}\right),
\end{aligned}
$$

substituting (58) and (61) in (55), we get

$$
\begin{aligned}
& \mathbb{H}^{\alpha}(t)\|u\|_{2}^{2} \leq c_{10}\left(\|v\|_{2(p+2)}^{2(p+2)}+\|u\|_{2(p+2)}^{2(p+2)}\right)+c_{10} \mathbb{H}(t), \\
& \mathbb{H}^{\alpha}(t)\|v\|_{2}^{2} \leq c_{11}\left(\|u\|_{2(p+2)}^{2(p+2)}+\|v\|_{2(p+2)}^{2(p+2)}\right)+c_{11} \mathbb{H}(t),
\end{aligned}
$$

Combining (53) and (63), using (31), we get

$$
\begin{aligned}
\mathscr{K}^{\prime}(t) \geq & {[(1-\alpha)-\varepsilon \kappa] \mathbb{H}^{-\alpha} \mathbb{H}^{\prime}(t) } \\
& +\varepsilon[(p+2)(1-\alpha)+1]\left(\left\|u_{t}\right\|_{2}^{2}+\left\|v_{t}\right\|_{2}^{2}+\|u\|_{2}^{2}+\|v\|_{2}^{2}\right) \\
& +\varepsilon\left\{[(p+2)(1-\alpha)-1]-\left(\int_{0}^{t} g(s) d s\right)\right. \\
& \left.\cdot\left[(p+2)(1-\alpha)-\frac{1}{2}\right]\right\}\|\nabla u\|_{2}^{2} \\
& +\varepsilon\left\{[(p+2)(1-\alpha)-1]-\left(\int_{0}^{t} h(s) d s\right)\right. \\
& \left.\cdot\left[(p+2)(1-\alpha)-\frac{1}{2}\right]\right\}\|\nabla v\|_{2}^{2} \\
& +\varepsilon(p+2)(1-\alpha) K(y, z) \\
& +\varepsilon\left[(p+2)(1-\alpha)-\frac{1}{2}\right](g o \nabla u+h o \nabla v) \\
& +\varepsilon\left(c_{0} a-\frac{\lambda_{1}+\lambda_{2}}{2 c_{3} \kappa}\right)\left[\|u\|_{2(p+2)}^{2(p+2)}+\|v\|_{2(p+2)}^{2(p+2)}\right] \\
& +\varepsilon\left(2(p+2)(1-\alpha)-\frac{\lambda_{1}+\lambda_{2}}{2 c_{3} \kappa}\right) \mathbb{H}(t),
\end{aligned}
$$

where $\lambda_{1}=c_{10} \int_{\tau_{1}}^{\tau_{2}}\left|\mu_{2}(\mathrm{\varrho})\right| d \mathrm{Q}, \lambda_{2}=c_{11} \int_{\tau_{1}}^{\tau_{2}}\left|\mu_{4}(\mathrm{\varrho})\right| d \mathrm{\varrho}$.

In this case, we take $a>0$ small enough, then

$$
\alpha_{1}=(p+2)(1-a)-1>0 \text {, }
$$

assuming

$$
\begin{aligned}
\max \left\{\int_{0}^{\infty} g(s) d s, \int_{0}^{\infty} h(s) d s\right\} & <\frac{(p+2)(1-a)-1}{((p+2)(1-a)-(1 / 2))} \\
& =\frac{2 \alpha_{1}}{2 \alpha_{1}+1},
\end{aligned}
$$


we have

$$
\begin{aligned}
& \left.\alpha_{2}=\{(p+2)(1-a)-1)-\int_{0}^{t} g(s) d s\left((p+2)(1-a)-\frac{1}{2}\right)\right\}>0, \\
& \left.\alpha_{3}=\{(p+2)(1-a)-1)-\int_{0}^{t} h(s) d s\left((p+2)(1-a)-\frac{1}{2}\right)\right\}>0,
\end{aligned}
$$

choose $\kappa$ so large that

$$
\begin{aligned}
& \alpha_{4}=a c_{0}-\frac{\lambda_{1}+\lambda_{2}}{2 c_{3} \kappa}>0, \\
& \alpha_{5}=2(p+2)(1-a)-\frac{\lambda_{1}+\lambda_{2}}{2 c_{3} \kappa}>0,
\end{aligned}
$$

fix $\kappa$ and $a$, we appoint $\varepsilon$ small enough so that

$$
\alpha_{6}=(1-\alpha)-\varepsilon \kappa>0
$$

Then, for $\beta>0$, we estimate (64) and it becomes

$$
\begin{aligned}
\mathscr{K}^{\prime}(t) \geq & \beta\left\{\mathbb{H}(t)+\left\|u_{t}\right\|_{2}^{2}+\left\|v_{t}\right\|_{2}^{2}+\|u\|_{2}^{2}+\|v\|_{2}^{2}+\|\nabla u\|_{2}^{2}\right. \\
& +\|\nabla v\|_{2}^{2}+(g o \nabla u)+(h o \nabla v)+K(y, z) \\
& \left.+\left[\|u\|_{2(p+2)}^{2(p+2)}+\|u\|_{2(p+2)}^{2(p+2)}\right]\right\} .
\end{aligned}
$$

By (31), for $\beta_{1}>0$, we get

$$
\begin{aligned}
\mathscr{K}^{\prime}(t) \geq & \beta_{1}\left\{\mathbb{H}(t)+\left\|u_{t}\right\|_{2}^{2}+\left\|v_{t}\right\|_{2}^{2}+\|u\|_{2}^{2}+\|v\|_{2}^{2}+\|\nabla u\|_{2}^{2}\right. \\
& +\|\nabla v\|_{2}^{2}+(g o \nabla u)+(h o \nabla v)+K(y, z) \\
& \left.+\left[\|u+v\|_{2(p+2)}^{2(p+2)}+2\|u v\|_{p+2}^{p+2}\right]\right\}
\end{aligned}
$$$$
\mathscr{K}(t) \geq \mathscr{K}(0)>0, t>0 .
$$

Using Holder's and Young's inequalities, we have

$$
\begin{aligned}
\left|\int_{\Omega}\left(u u_{t}+v v_{t}\right) d x\right|^{1 /(1-\alpha)} \geq & C\left[\|u\|_{2(p+2)}^{\theta /(1-\alpha)}+\left\|u_{t}\right\|_{2}^{\mu /(1-\alpha)}\right. \\
& \left.+\|v\|_{2(p+2)}^{\theta /(1-\alpha)}+\left\|v_{t}\right\|_{2}^{\mu /(1-\alpha)}\right],
\end{aligned}
$$

where $(1 / \mu)+(1 / \theta)=1$ put $\theta=2(1-\alpha)$, to get

$$
\frac{\mu}{1-\alpha}=\frac{2}{1-2 \alpha} \leq 2(p+2)
$$

Subsequently, for $s=2 /(1-2 \alpha)$ and by using (39), we get

$$
\begin{aligned}
& \|u\|_{2(p+2)}^{2 /(1-2 \alpha)} \leq d\left(\|u\|_{2(p+2)}^{2(p+2)}+\mathbb{H}(t)\right), \\
& \|v\|_{2(p+2)}^{2 /(1-2 \alpha)} \leq d\left(\|v\|_{2(p+2)}^{2(p+2)}+\mathbb{H}(t)\right), \quad \forall t \geq 0 .
\end{aligned}
$$

Therefore,

$$
\begin{aligned}
& \left|\int_{\Omega}\left(u u_{t}+v v_{t}\right) d x\right|^{1 /(1-\alpha)} \\
& \quad \geq c_{12}\left[\|u\|_{2(p+2)}^{2(p+2)}+\|v\|_{2(p+2)}^{2(p+2)}+\left\|u_{t}\right\|_{2}^{2}+\left\|v_{t}\right\|_{2}^{2}+\mathbb{H}(t)\right] .
\end{aligned}
$$

Subsequently,

$$
\begin{aligned}
\mathscr{K}^{1 /(1-\alpha)}(t)= & \mathbb{H}^{1-\alpha}+\varepsilon \int_{\Omega}\left(u u_{t}+v v_{t}\right) d x \\
& +\frac{\varepsilon}{2} \int_{\Omega}\left(\mu_{1} u^{2}+\mu_{3} v^{2}\right) d x \\
& \left.+\frac{\varepsilon}{2} \int_{\Omega}\left(\omega_{1} \nabla u^{2}+\omega_{2} \nabla v^{2}\right) d x\right)^{1 /(1-\alpha)} \\
\leq & c\left\{\mathbb{H}(t)\left|\int_{\Omega}\left(u u_{t}+v v_{t}\right) d x\right|^{1 /(1-\alpha)}+\|u\|_{2}^{2 /(1-\alpha)}\right. \\
& \left.+\|\nabla u\|_{2}^{2 /(1-\alpha)}+\|v\|_{2}^{2 /(1-\alpha)}+\|\nabla v\|_{2}^{2 /(1-\alpha)}\right\} \\
\leq & c\left[\mathbb{H}(t)+\left\|u_{t}\right\|_{2}^{2}+\left\|v_{t}\right\|_{2}^{2}+\|u\|_{2}^{2}+\|v\|_{2}^{2}\right. \\
& +\|\nabla u\|_{2}^{2}+\|\nabla v\|_{2}^{2}+(g o \nabla u)+(h o \nabla v) \\
& \left.+\|u\|_{2(p+2)}^{2(p+2)}+\|v\|_{2(p+2)}^{2(p+2)}\right]
\end{aligned}
$$

From (70) and (76), gives

$$
\mathscr{K}^{\prime}(t) \geq \lambda \mathscr{K}^{\frac{1}{1-\alpha}}(t)
$$

with $\lambda>0$, this quantity depends on $\beta$ and $c$. By simple integration of (77), we obtain

$$
\mathscr{K}^{\alpha /(1-\alpha)}(t) \geq \frac{1}{\mathscr{K}^{-\alpha /(1-\alpha)}(0)-\lambda(\alpha /(1-\alpha)) t},
$$

Hence, $\mathscr{K}(t)$ in a situation of blow up in time, when

$$
T \leq T^{*}=\frac{1-\alpha}{\lambda \alpha \mathscr{K}^{\alpha} /^{(1-\alpha)}(0)},
$$

Then, this completes the proof of the theorem. 


\section{Conclusion}

In this work, we have studied the blow up of the coupled Klein-Gordon system with strong damping, distributed delay, and source terms, under suitable conditions which are so important that we find them in many applications of natural sciences. Many authors have been concerned with this problem in recent decades (see, for example, [17-19]). In the next work, we will try to apply the same technique with a new class of Boussinesq equations which are nonlinear partial differential equation that arises in hydrodynamics and some physical applications. It was subsequently applied to problems in the percolation of water in porous subsurface strata (see, for example, $[20,21]$ ).

\section{Data Availability}

No data were used to support this study.

\section{Conflicts of Interest}

The authors declare that there is no conflict of interests regarding the publication of this manuscript. The authors declare that they have no competing interests.

\section{Authors' Contributions}

The authors contributed equally in this article. They have all read and approved the final manuscript.

\section{References}

[1] S. Berrimi and S. Messaoudi, "Existence and decay of solutions of a viscoelastic equation with a nonlinear source," Nonlinear Analysis, vol. 64, no. 10, pp. 2314-2331, 2006.

[2] M. M. Cavalcanti, D. Cavalcanti, and J. Ferreira, "Existence and uniform decay for a non-linear viscoelastic equation with strong damping," Mathematical Methods in the Applied Sciences, vol. 24, no. 14, pp. 1043-1053, 2001.

[3] M. M. Cavalcanti, D. Cavalcanti, P. J. S. Filho, and J. A. Soriano, "Existence and uniform decay rates for viscoelastic problems with nonlinear boundary damping," Differential and Integral Equations, vol. 14, pp. 85-116, 2001.

[4] M. Kafini and S. A. Messaoudi, "A blow-up result in a Cauchy viscoelastic problem," Applied Mathematics Letters, vol. 21, no. 6, pp. 549-553, 2008.

[5] H. T. Song and D. S. Xue, "Blow up in a nonlinear viscoelastic wave equation with strong damping," Nonlinear Analysis: Theory, Methods \& Applications, vol. 109, pp. 245-251, 2014.

[6] H. T. Song and C. K. Zhong, "Blow-up of solutions of a nonlinear viscoelastic wave equation," Nonlinear Analysis: Real World Applications, vol. 11, no. 5, pp. 3877-3883, 2010.

[7] K. Zennir, "Exponential growth of solutions with $L_{p}$-norm of a nonlinear viscoelastic hyperbolic equation," Journal of Nonlinear Sciences \& Applications, vol. 6, no. 4, pp. 252-262, 2013.

[8] L. Guo, Z. Yuan, and G. Lin, "Blow up and global existence for a nonlinear viscoelastic wave equation with strong damping and nonlinear damping and source terms," Applied Mathematics, vol. 6, no. 5, pp. 806-816, 2015.
[9] K. Agre and M. A. Rammaha, "Systems of nonlinear wave equations with damping and source terms," Differential and Integral Equations, vol. 19, pp. 1235-1270, 2007.

[10] V. Georgiev and G. Todorova, "Existence of a solution of the wave equation with nonlinear damping and source terms," Journal of Differential Equations, vol. 109, no. 2, pp. 295308, 1994.

[11] A. Ben Aissa, D. Ouchenane, and K. Zennir, "Blow up of positive initial-energy solutions to systems of nonlinear wave equations with degenerate damping and source terms," Nonlinear Studies, vol. 4, pp. 523-535, 2012.

[12] D. Ouchenane, K. Zennir, and M. Bayoud, "Global nonexistence of solutions for a system of nonlinear viscoelastic wave equations with degenerate damping and source terms," Ukrainian Mathematical Journal, vol. 65, no. 5, pp. 723-739, 2013.

[13] S. Nicaise and C. Pignotti, "Stabilization of the wave equation with boundary or internal distributed delay," Differential and Integral Equations, vol. 21, no. 9-10, pp. 935-958, 2008.

[14] M. A. Ragusa and A. Tachikawa, "Regularity of minimizers of some variational integrals with discontinuity," Zeitschrift fur Analysis und ihre Anwendungen, vol. 27, no. 4, pp. 469-482, 2008.

[15] M. A. Ragusa and A. Tachikawa, "Regularity for minimizers for functionals of double phase with variable exponents," Advances in Nonlinear Analysis, vol. 9, pp. 710-728, 2019.

[16] L. H. K. Son, L. T. P. Ngoc, and N. T. Long, "Existence, blowup and exponential decay estimates for the nonlinear Kirchhoffff-carrier wave equation in an annular with nonhomogeneous Dirichlet conditions," Filomat, vol. 33, no. 17, pp. 5561-5588, 2019.

[17] S. Boulaaras, A. Choucha, D. Ouchenane, and B. Cherif, "Blow up of solutions of two singular nonlinear viscoelastic equations with general source and localized frictional damping terms," Advances in Difference Equations, vol. 2020, no. 1, 2020.

[18] A. Rahmoune, D. Ouchenane, S. Boulaaras, and P. Agarwal, "Growth of solutions for a coupled nonlinear Klein-Gordon system with strong damping, source, and distributed delay terms," Advances in Difference Equations, vol. 2020, no. 1, 2020.

[19] A. Zarai, A. Draifia, and S. Boulaaras, "Blow up of solutions for a system of nonlocal singular viscoelatic equations," Applicable Analysis, vol. 97, no. 13, pp. 2231-2245, 2018.

[20] R. P. Agarwal, S. Gala, and M. A. Ragusa, "A regularity criterion in weak spaces to Boussinesq equations," Mathematics, vol. 8, no. 6, p. 920, 2020.

[21] A. Barbagallo, S. Gala, M. A. Ragusa, and M. Théra, "On the regularity of weak solutions of the Boussinesq equations in Besov spaces," Vietnam Journal of Mathematics, 2020. 